\title{
Electric Dichroism and Bending Amplitudes of DNA Fragments According to a Simple Orientation Function for Weakly Bent Rods
}

\author{
DIETMAR PORSCHKE, Max-Planck Institut für biophysikalische \\ Chemie, D-3400 Göttingen, Federal Republic of Germany
}

\begin{abstract}
Synopsis
The linear dichroism is calculated for DNA fragments in their thermal bending equilibrium. These calculations are given for relatively short fragments, where bent molecules can be described by an arc model. Using the measured value of $350 \AA$ for the persistence length, the limit dichroism (corresponding to complete alignment) decreases due to thermal bending, e.g., for a fragment with 100 base pairs to $80 \%$ of the value expected for straight molecules. Thermal bending should lead to a strong continuous decrease of the dichroism with increasing chain length, which is not observed, however, in electric dichroism experiments due to electric stretching. The influence of the electric field on the bending equilibrium is described by a contribution to the bending energy, which is calculated from the movement of charge equivalents against the potential gradient upon bending. The charge equivalents, which are assigned to the helix ends, are derived from the dipole moments causing the stationary degree of orientation. By this procedure the energy term inducing DNA stretching is given for induced, permanent, and saturating induced dipole models without introduction of any additional parameter. The stationary dichroism at a given electric field strength is then calculated according to an arc model by integration over all angles of orientation of helix axes or chords with respect to the field vector, and at each of these angles the contribution to the dichroism is calculated by integration over all helices with different degrees of bending. Orientation functions obtained by this procedure are fitted to dichroism data measured for various restriction fragments. Optimal fits are found for an induced dipole model with saturation of the polarizability. The difference between orientation functions with and without electric stretching is used to evaluate dichroism bending amplitudes. Both chain length and field strength dependence of bending amplitudes are consistent with experimental amplitudes derived from the dichroism decay in low salt buffers containing multivalent ions like $\mathrm{Mg}^{2+}$, spermine, or $\left.\left[\mathrm{CoNH}_{3}\right)_{6}\right]^{3+}$. Bending amplitudes can be used to evaluate the persistence length from electrooptical data obtained for a single DNA restriction fragment. Bending and stretching effects are considerable already at relatively low chain length, and thus should not be neglected in any quantitative evaluation of experimental data.
\end{abstract}

\section{INTRODUCTION}

Dichroism measurements provide very useful information on structures of macromolecules in solution..$^{1-3} \mathrm{~A}$ qualitative interpretation of the dichroism data, however, requires appropriate models, which are not yet available for all cases of practical interest. The stationary level of the dichroism measured as a function of the electrical field strength has been discussed with special emphasis on the nature of electrical properties ${ }^{4,5}$ - usually under the assumption of rigid molecular structures. However, most macromolecules are flexible - at least to some extent-and this flexibility may have a considerable influence on the experimental results. DNA double helices, for example, can be regarded as almost rigid structures in the limit of low chain lengths, but their 
properties in solution are clearly influenced by their flexibility ${ }^{6-9}$ at chain lengths $\geq 100$ base pairs (bp). Obviously, the flexibility should be considered in the evaluation of dichroism measurements in order to get more accurate information on the molecules in solution. In the present contribution a relatively simple model is described, which has been developed for an analysis of DNA structure in solution. The same concept should be useful for analysis of other flexible macromolecules.

\section{FACTORS CONTRIBUTING TO THE ORIENTATION FUNCTION OF FLEXIBLE RODS}

\section{Thermal Bending Equilibrium}

The bending equilibrium of flexible rods has been discussed in textbooks ${ }^{10,11}$ and has been applied to describe the properties of DNA molecules in solution. ${ }^{11,12}$ Thus, for the present purpose a short summary of some results, which are used to derive the orientation function, should be sufficient. When a rod of a contour length $\mathscr{L}$ is bent at a bending angle $\Theta$, the free energy of bending is given by

$$
\Delta G_{m}=\frac{\sigma}{2 \mathscr{L}} \cdot \Theta^{2}
$$

where $\sigma$ is the mechanical bending constant and $\Theta$ is the angle between tangents to the end of the rod. Bending is assumed to be uniform.

Because a rod can be bent in two directions with mechanical bending constants $\sigma_{1}$ and $\sigma_{2}$, which need not be equivalent, and because bending in the two directions with the bending angles $\Theta_{1}$ and $\Theta_{2}$ is independent, the free energy in the general case is given by

$$
\Delta G_{m}=\frac{\sigma_{1} \cdot \Theta_{1}^{2}}{2 \mathscr{L}}+\frac{\sigma_{2} \cdot \Theta_{2}^{2}}{2 \mathscr{L}}
$$

For small degrees of bending, the overall bending angle $\Theta$ is related to the individual ones by

$$
\Theta^{2}=\Theta_{1}^{2}+\Theta_{2}^{2}
$$

The mean square bending angle for the distribution of bent rods has to be calculated by integration over all combinations of $\Theta_{1}$ and $\Theta_{2}$ according to

$$
\begin{aligned}
\left\langle\Theta^{2}\right\rangle & =\frac{\int_{-\infty}^{\infty} \int_{-\infty}^{\infty} \Theta^{2} \cdot e^{-\Delta G_{m}\left(\Theta_{1}, \Theta_{2}\right) / k T} \cdot d \Theta_{1} d \Theta_{2}}{\int_{-\infty}^{\infty} \int_{-\infty}^{\infty} e^{-\Delta G_{m}\left(\Theta_{1}, \Theta_{2}\right) / k T} \cdot d \Theta_{1} \cdot d \Theta_{2}} \\
& =\mathscr{L} \cdot k T\left(\frac{1}{\sigma_{1}}+\frac{1}{\sigma_{2}}\right)
\end{aligned}
$$

The mechanical bending constant $\sigma$, which does not depend on the bending direction in the case of DNA $\left(\sigma=\sigma_{1}=\sigma_{2}\right)$, may be calculated from literature 
values $^{7-9,13-15}$ of the persistence length $p$ according to

$$
\sigma=k T \cdot p
$$

The persistence length of DNA has been evaluated, for example, from rotation diffusion coefficients of DNA helices with various, exactly defined chain lengths. According to the experimental value valid at high degrees of electrostatic shielding (high salt or presence of multivalent ions ${ }^{13-15}$ ) $p=3.5 \cdot 10^{-8} \mathrm{~m}$ ( $\approx 100 \mathrm{bp}$ ), the square root of $\left\langle\Theta^{2}\right\rangle$ is 1.4 (in radians) for helices with $100 \mathrm{bp}$, which corresponds to a considerable average degree of bending.

\section{Dichroism of Curved Molecules}

Since the linear dichroism depends on the positions of the transition dipole moments, its magnitude should be affected by bending. Although the linear dichroism may be calculated for any defined arrangement of chromophores, convenient calculations require chromophores, which are arranged at a relatively simple geometry. For bending of rodlike molecules it is reasonable to assume that bending is uniform and that the chromophores are arranged according to a circular arc. The linear dichroism for this model, which corresponds exactly to the mechanical one described above, has been evaluated previously. It is given in the form of the reduced limit dichroism defined by

$$
\frac{\Delta \epsilon^{\infty}}{\epsilon}=\frac{\Delta \epsilon_{\|}^{\infty}-\Delta \epsilon_{\perp}^{\infty}}{\epsilon_{r}}
$$

where $\Delta \epsilon_{\|}^{\infty}$ and $\Delta \epsilon_{\perp}^{\infty}$ are the changes of the extinction coefficient in the limit of complete orientation (at infinite field strength) observed with light parallel and perpendicular to the field vector, respectively. $\epsilon_{r}$ denotes the extinction coefficient at random distribution. For a circular arc the reduced limit dichroism is given by

$$
\begin{aligned}
\frac{\Delta \epsilon^{\infty}}{\epsilon}=\frac{3}{2}\left\{3 \cdot \frac{1}{\varphi}\right. & {\left[\cos ^{2} \kappa\left(\frac{1}{2} \sin \varphi \cdot \cos \varphi+\frac{\varphi}{2}\right)\right.} \\
& \left.\left.+\frac{1}{2}\left(1-\cos ^{2} \kappa\right)\left(-\frac{1}{2} \sin \varphi \cdot \cos \varphi+\frac{\varphi}{2}\right)\right]-1\right\}
\end{aligned}
$$

where $\varphi$ is half of the complete arc angle $\Theta$ and $\kappa$ defines the angle of the transition dipoles with respect to the axis of the straight rod (for details of the evaluation, cf. Ref. 13).

\section{Average Dichroism of DNA Under Thermal Bending}

By combination of equations for thermal bending of double helices and for their dichroism, it is now possible to calculate the average dichroism in thermal equilibrium according to

$$
\frac{\overline{\Delta \epsilon}}{\epsilon}=\frac{\int_{-\infty}^{\infty} \int_{-\infty}^{\infty} \frac{\Delta \epsilon}{\epsilon}(\Theta) \cdot e^{-\Delta G_{m}\left(\Theta_{1}, \Theta_{2}\right) / k T} \cdot d \Theta_{1} d \Theta_{2}}{\int_{-\infty}^{\infty} \int_{-\infty}^{\infty} e^{-\Delta G_{m}\left(\Theta_{1}, \Theta_{2}\right) / k T} \cdot d \Theta_{1} \cdot d \Theta_{2}}
$$




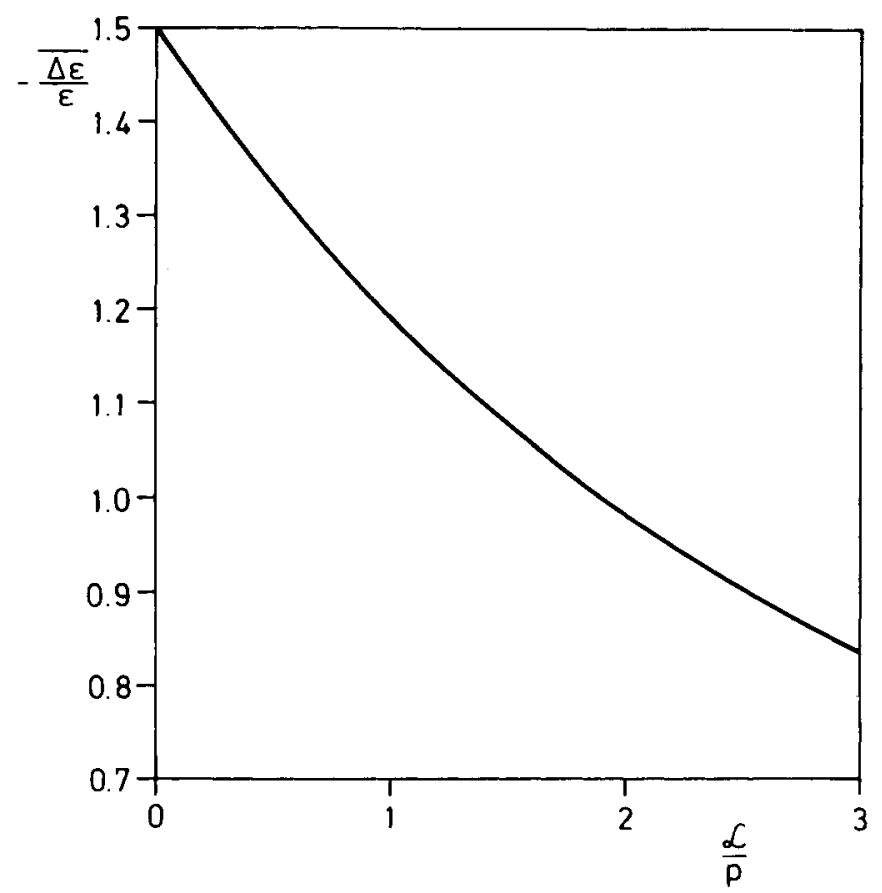

Fig. 1. Average dichroism $\overline{\Delta \epsilon} / \epsilon$ for the distribution of bent double helices in their thermal bending equilibrium as a function of the contour length $\mathscr{L}$ relative to the persistence length $p$ for perpendicular alignment of transition dipoles to the helix axis $(\kappa=\pi / 2)$.

This value applies for the limit of complete alignment of the helices with their axis or chord parallel to the field vector. For sterical reasons, helices cannot be bent up to a bending angle $\infty$ but only to a circular form, which corresponds to a bending angle $\Theta=2 \pi(\varphi=\pi)$. A numerical calculation with this boundary condition, with the experimental persistence length $p=3.5 \cdot 10^{-8} \mathrm{~m}$ and with a perpendicular alignment of the transition dipoles for the helix axis $(\kappa=\pi / 2)$ provides average dichroism values of -1.210 for a helix with $95 \mathrm{bp}$ and -1.028 for a helix with $179 \mathrm{bp}$. Upon complete stretching the dichroism of these helices would be -1.5 . Thus, thermal bending strongly affects the dichroism and should not be neglected in quantitative evaluations of experimental data.

As shown in Fig. 1, thermal bending should lead to a continuous decrease of the dichroism with increasing chain length. Because this prediction is not consistent with experimental values, ${ }^{5}$ the experimental values of the dichroism are apparently influenced by still another effect.

\section{Stretching Due to Dipole Forces in an Electric Field}

Electric fields induce a polarization of the ion atmosphere $\mathrm{r}^{16-19}$ around the DNA, which leads to effective excess charges at the ends of the double helix. These excess charges do not only cause orientation but also stretching of bent helices. At present a complete theory of DNA polarization is not available on an appropriate molecular basis. Thus it is also difficult to design an exact theory of the DNA stretching force. 
However, the stretching effect may be described reasonably well on a simple phenomenological level. This may be justified by the fact that the parallel process of DNA orientation is generally described in the literature on a merely phenomenological level as well. Evaluation of the orientational distribution according to orientation functions with induced or permanent dipoles provides parameters of the dipolar forces, which are experienced by the double helices. It should be possible to describe the stretching forces by a corresponding model. The dipole moment $\mu$ of a molecule with opposite charges $q$ at a distance $d$ is defined as

$$
\mu=q \cdot d
$$

According to this equation, a dipole $\mu$ observed for a DNA molecule may be represented by two opposite charges at the ends of the molecule. Since the length of the DNA is defined by the number of base pairs $n$ and the length increment per base pair $\delta=3.4 \cdot 10^{-10} \mathrm{~m}$ (valid for the B-form of DNA), ${ }^{20}$ the effective charge $q_{e}$ at the end of the double helix is

$$
q_{e}=\frac{\mu}{n \cdot \delta}
$$

Of course, this model does not reflect the true molecular distribution of charges around the double helix, but it reflects exactly the torque experienced by the DNA in the electric field and thus should also be a reasonable basis for a treatment of the stretching process.

The energy required for bending of a straight molecule, which arises in the presence of an electric field and has to be provided in addition to the mechanical energy discussed above, can be easily calculated on the basis of the excess charges located at the helix ends. For this calculation we start with a DNA molecule aligned parallel to the field vector. Bending is again assumed to be uniform in one plane, such that the axis of the DNA forms a circular arc with its chord parallel to the field vector. In this case the excess charges at each end of the molecule have to be moved by

$$
\Delta s=\frac{n \cdot \delta}{2}\left(1-\frac{\sin \varphi}{\varphi}\right)
$$

against the potential gradient upon bending at a half arc angle $\varphi$ (cf. Fig. 2). Movement of excess charges $q_{e}$ against the potential gradient requires a total amount of "electrical" energy for DNA bending

$$
\Delta G_{e}=n \cdot \delta\left(1-\frac{\sin \varphi}{\varphi}\right) \cdot q_{e} E_{e}
$$

where $E_{e}$ denotes the effective electric field strength. If the axis of the DNA or the chord of a DNA arc forms an angle $\vartheta$ with respect to the field vector, the component

$$
E_{e}=E_{0} \cos \vartheta
$$

of the applied electric field $E_{0}$ is used for the calculation. 


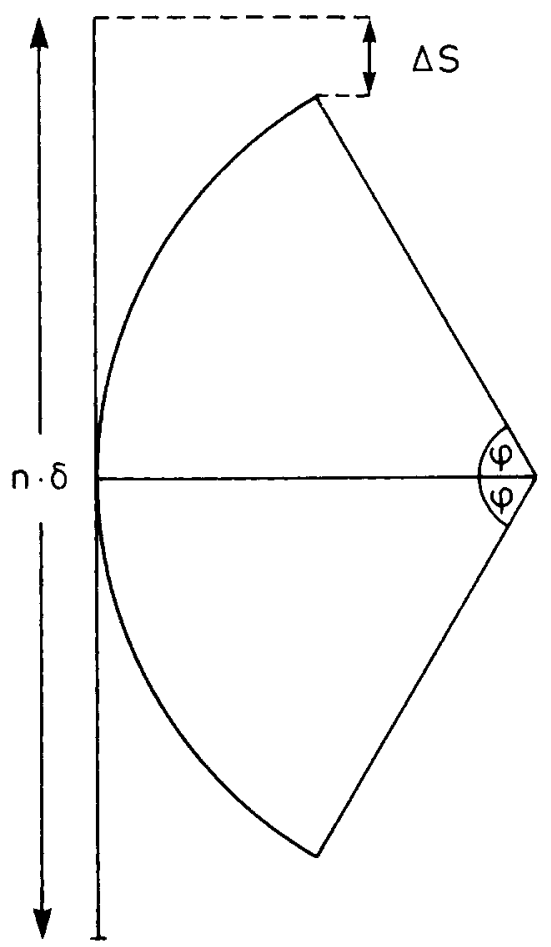

Fig. 2. Schematic representation of bending a rod with a contour length $\mathscr{L}=n \cdot \delta$ to a bending angle $\Theta=2 \varphi$. Bending requires that the helix ends are moved by $\Delta s$ against the potential gradient.

\section{Coupling of Orientation and Stretching}

Upon application of an electric field the isotropic distribution of molecules is replaced by a new distribution with a preferential direction parallel to the field vector. The field-induced distribution is calculated as a Boltzmann distribution defined by the energy of dipoles at the various angles of orientation with respect to the field vector (cf. Refs. 1 and 2). In the present case, integration also includes the distribution of molecules with different degrees of bending and, furthermore, the influence of the electric field on this distribution. Obviously the stretching effect due to the electric field is dependent upon the angle of molecular orientation, which is described by using the component of the field vector in the direction of the helix axis for the calculation [cf. Eq. (13)]. In the case of bent molecules the orientation with respect to the field vector will be defined by the chord of the arc. For any given angle of the chord relative to the field vector, the distribution of bent molecules is assumed to be isotropic with respect to rotation around the chord. This assumption implies that the components of the polarizability or of the dipole moment are not considered completely - an approximation, which is common to all previously applied models of orientation. The error resulting from this approximation may be neglected at moderate degrees of bending, whereas results for strongly bent molecules should be interpreted with caution. ${ }^{21} \mathrm{~A}$ further approximation is used with respect to the magnitude of the polarizability and of the dipole 
moments: in order to limit the complexity of the calculations, these magnitudes are assumed to be independent of DNA bending.

The distribution of DNA molecules is calculated according to the following procedure: Bending of DNA in the presence of an electric field requires mechanical and electrical energy as described above

$$
\Delta G_{b}=\Delta G_{m}+\Delta G_{e}
$$

and the distribution of bent molecules at a given alignment angle $\vartheta$ is described by the equations given in the section on thermal bending equilibrium.

At each bending angle $\Theta=2 \varphi$ the dichroism is defined by Eq. (7), which is used to calculate the mean value of the dichroism at any given angle of orientation $\vartheta$ according to

$$
\frac{\overline{\Delta \epsilon}}{\epsilon}(\vartheta)=\frac{\int_{-\infty}^{\infty} \int_{-\infty}^{\infty} e^{-\Delta G_{b}^{\vartheta} / k T} \cdot \frac{\Delta \epsilon}{\epsilon}(\Theta) \cdot d \Theta_{1} d \Theta_{2}}{\int_{-\infty}^{\infty} \int_{-\infty}^{\infty} e^{-\Delta G_{b}^{\vartheta} / k T} d \Theta_{1} d \Theta_{2}}
$$

Finally, the dichroism has to be integrated over all possible angles of orientation

$$
\frac{\Delta \epsilon}{\epsilon}=\frac{\int_{0}^{\pi} e^{-U / k T} \cdot \frac{\overline{\Delta \epsilon}}{\epsilon}(\vartheta) \cdot \frac{3 \cos ^{2} \vartheta-1}{2} \cdot 2 \pi \sin \vartheta d \vartheta}{\int_{0}^{\pi} e^{-U / k T} \cdot 2 \pi \sin \vartheta d \vartheta}
$$

where $U$ is the energy of the induced or permanent dipole at a given angle $\vartheta$ with respect to the field vector.

\section{Comparison with Experimental Data}

The orientation function described in the previous section is rather complex and thus has been solved by numerical integration. The integration procedure may be simplified by introduction of polar coordinates, but the present form can be modified more easily to include stable curvature. The bending equilibrium has not been integrated from bending angles of $-\infty$ to $+\infty$, but for sterical recsons (cf. above) and because of symmetry only from $\varphi=0$ to $\varphi=\pi$. The limit angle $\varphi=\pi$ corresponds to circular forms of the DNA fragments. Appropriate dipole terms have been introduced for the cases of induced, permanent, or saturating induced dipole moments. ${ }^{5,22,23}$ The whole integration procedure has been coupled to a least-squares fitting routine for the evaluation of optimal model parameters from experimental dichroism data.

The experimental procedures and the DNA fragments used for the experiments have been described. ${ }^{5,13}$ Nevertheless, a few general comments may be useful. The DNA fragments, which have been generated by digestion of plasmid DNA with HaeIII restriction nuclease, are blunt ended and their migration in gel electrophoresis does not deviate from standards. The stationary dichroism of these fragments has been evaluated from the differences of 
the stationary electrooptical signals in the absence and the presence of electric fields; the electric field pulses were sufficiently long to induce stationary degrees of orientation. The persistence lengths used for the evaluations have been determined previously ${ }^{13}$ from rotational diffusion time constants according to the weakly bending rod model developed by Hearst. ${ }^{24}$ Since these time constants and the stationary dichroism data analysed below have been measured for the same fragments and in the same buffers, all these data should be consistent. The standard temperature used for all measurements was $20^{\circ} \mathrm{C}$.

The experimental data obtained for short DNA fragments ( $\leq 69 \mathrm{bp}$ ) could be fitted by an induced dipole model and not by a permanent dipole model. Conversely, data obtained for long fragments ( $\geq 256 \mathrm{bp}$ ) could be fitted by a permanent and not by an induced dipole model. In the intermediate range of chain lengths, a transition from an induced to a permanent dipole character is observed. These details have been characterized previously and led to the development of a saturating induced dipole model. ${ }^{5}$ This model has been used for the present orientation function in the form of the square root approximation. ${ }^{22,23}$ All the dichroism data in the range of chain lengths from 42 to 256 bp and field strengths from 2 to $70 \mathrm{kV} / \mathrm{cm}$ could be represented by this model within experimental accuracy. An example is given in Fig. 3.

The new orientation function cannot only be used to evaluate the stationary dichroism measured as a function of the electric field strength, but it can also provide information on the extent of field-induced stretching of DNA helices. The reverse process of DNA bending is observed after pulse termination $^{9,13}$ and is reflected by a relaxation component in the dichroism decay curves, which is much faster than the overall "tumbling" relaxation (cf. Fig. 4). The present orientation function does not describe the rotational diffusion after pulse termination, but only the stationary degree of the dichroism. Nevertheless, the orientation function gives information about the amplitudes of the decay processes provided that the bending distribution is equilibrated much more rapidly than the overall distribution of the molecules in space. Since diffusional rotation is very strongly dependent upon chain length, this condition is fulfilled sufficiently well. The dichroism amplitude due to bending may be evaluated by comparison of the stationary dichroism at a given field strength with and without field-induced stretching. A comparison of calculated and experimental bending amplitudes given in Table I as a function of the chain length at maximal experimental field strengths reveals a surprisingly close agreement in most cases investigated. A similar agreement is found for the dependence of the stretching/bending process on the electric field strength (cf. Fig. 3).

The calculated data are in close agreement with the experimental data obtained in the buffers containing $100 \mu M \mathrm{Mg}^{2+}$ or $3 \mu M$ spermine or $10 \mu M$ $\left[\mathrm{Co}\left(\mathrm{NH}_{3}\right)_{6}\right]^{3+}$ in addition to $1 \mathrm{mM} \mathrm{NaCl}$ and $1 \mathrm{mM}$ Na-cacodylate, $\mathrm{pH} 7.0$. However, the agreement is not satisfactory with respect to bending amplitudes observed in a buffer containing $100 \mu M$ EDTA, $1 \mathrm{mM} \mathrm{NaCl}$ and $1 \mathrm{mM}$ Na-cacodylate, $\mathrm{pH}$ 7.0. The experimental bending amplitudes found in this case are almost a factor of 2 higher than those calculated with the persistence length of $600 \AA$ obtained for this buffer from rotation relaxation time constants. The reason for the disagreement is not clear; some possible explanations are discussed in the following section. 


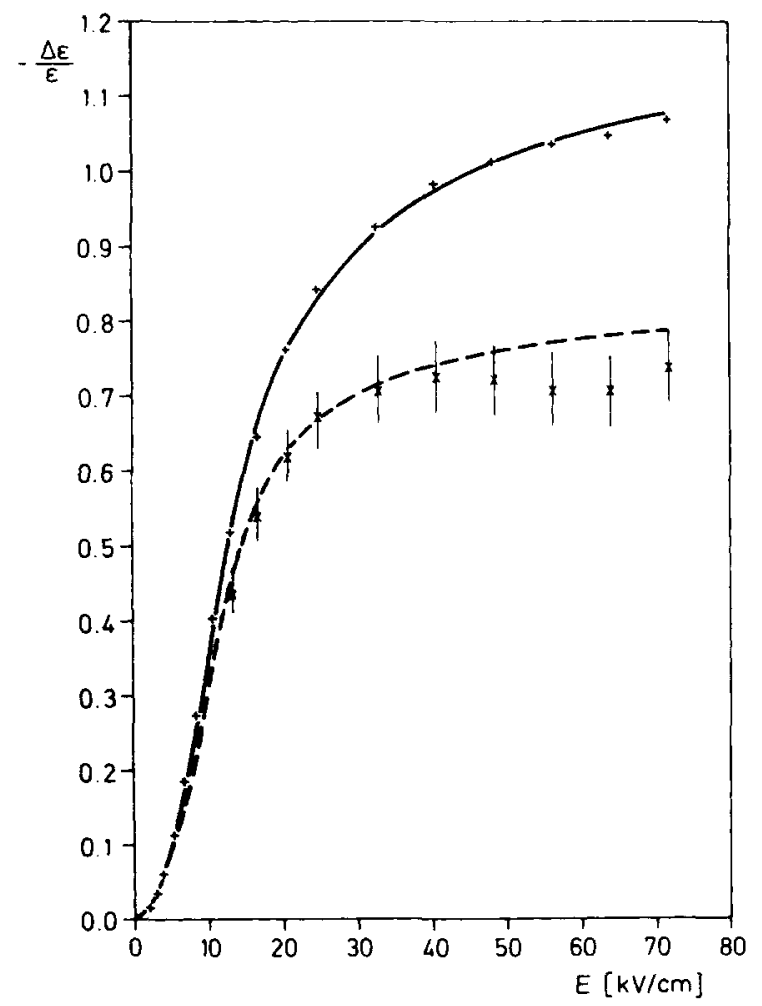

Fig. 3. Reduced linear dichroism $\Delta \epsilon / \epsilon$ as a function of the electric field strength $E$ for a DNA fragment with $179 \mathrm{bp}$ in $1 \mathrm{~m} M \mathrm{NaCl}, 1 \mathrm{~m} M \mathrm{Na}$-cacodylate, $\mathrm{pH} 7.0,100 \mu M \mathrm{Mg}^{2+}$ at $20^{\circ} \mathrm{C}$. The orientation function for flexible rods (-, cf. text) has been fitted to the experimental stationary dichroism $(+)$. The dashed line shows the slow dichroism decay amplitude predicted by the model; the corresponding experimental data $(x)$ are shown with estimated error bars.

A potential application of the new orientation function is the determination of the persistence length from electrooptical data obtained for single DNA fragments. This procedure provides persistence lengths, which are in agreement with those evaluated from series of rotation time constants according to the weakly bending rod model within experimental accuracy for the buffers containing $\mathrm{Mg}^{2+}$, spermine, or $\left[\mathrm{Co}\left(\mathrm{NH}_{3}\right)_{6}\right]^{3+}$. The bending amplitudes measured in the buffer without multivalent ions (cf. above) suggest a persistence length of about $100 \mathrm{bp}$, which is in contrast to the value derived from rotation time constants.

\section{DISCUSSION}

Although the flexibility of DNA double helices has been recognized and determined quantitatively a long time ago, DNA fragments have been described as rigid rods in all previous evaluations of the stationary dichroism or birefringence. As shown by the calculations given above, this simplification may lead to erroneous assignments of model parameters. The flexibility of double helices is represented by the present orientation function in the limit of relatively low chain lengths, where bent molecules may be described 

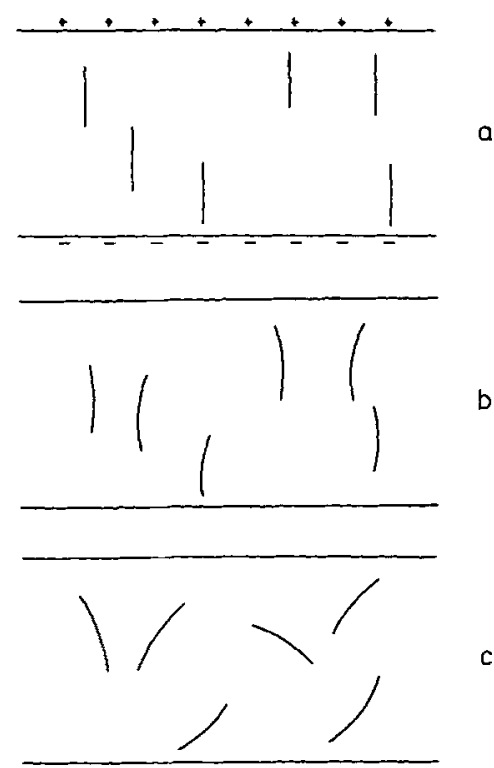

Fig. 4. Schematic representation of the processes observed after pulse termination. (a) In the presence of the electric field the molecules are aligned and stretched. (b) After pulse termination the molecules first return to their equilibrium distribution of bent states, since diffusional motion of helix ends is fast compared to overall rotation. This process is associated with a decrease of the linear dichroism. (c) Due to the strong chain length dependence of rotational diffusion, overall distribution of molecules in space is a relatively slow process.

reasonably well by an arc model. Since the dichroism of helical arcs has been calculated previously, ${ }^{13}$ the present task mainly was the appropriate combination with the statistical thermodynamic description of the bending distribution $^{10,11}$ and the design of a reasonable electrical stretching function. Stretching of a polyelectrolyte by an electric field is a very complex process, which is rather difficult to describe on a proper molecular basis. Of course, this is also true for the field-induced orientation of polyelectrolytes. Nevertheless, the orientation has always been described by simple empirical dipole models and thus stretching may be described on an empirical level as well. The present stretching function was selected partly because of its simplicity, but mainly because it is consistent with the dipole function used for the description of the orientation process and because it does not require the introduction of any new parameter. The resulting orientation function is clearly more complex than any function applied in the past for the analysis of electrooptical data and yet it reflects the complexity of molecular structure on a still relatively simple level.

Among the approximations, the most severe one appears to be the restriction of DNA bending to a circular arc. Obviously DNA molecules are free to bend at any site into any direction and thus the bending pattern is more complex (for a review, cf. Ref. 25). However, for the present purpose the exact pathway of the bent DNA chain is not as important as it may appear at first. The stationary value of the dichroism is determined by the average orientation of the chromophores with respect to the field vector. In this respect the arc model is expected to be a very reasonable approximation for relatively 
TABLE I

Parameters Obtained from Least-Squares Fitting of the Stationary Electric Dichroism to the Orientation Function for Weakly Bent Rods-with Saturation of the Polarizability ${ }^{5}$ According to the Square Root Approximation. ${ }^{20, \text { a }}$

\begin{tabular}{|c|c|c|c|c|c|c|c|}
\hline & Buffer & 43 & 69 & 84 & 95 & 179 & 256 \\
\hline \multirow[t]{4}{*}{$\alpha$} & A & 1.32 & 3.79 & 5.80 & 7.26 & 26.0 & 48.6 \\
\hline & B & 1.43 & 4.07 & 6.13 & 7.60 & 31.6 & 63.5 \\
\hline & $\mathrm{C}$ & 1.23 & 2.96 & 4.05 & 6.38 & 19.3 & 46.8 \\
\hline & D & 1.34 & 3.95 & 6.00 & 7.47 & 24.5 & 28.4 \\
\hline \multirow[t]{4}{*}{$E_{0}$} & A & 84 & 47 & 36 & 24.8 & 8.7 & 4.4 \\
\hline & B & $>100$ & 55 & 38 & 27.2 & 11.1 & 8.9 \\
\hline & $\mathrm{C}$ & 67 & 35 & 24 & 18.7 & 13.6 & 6.5 \\
\hline & D & 52 & 41 & 29 & 24.4 & 8.0 & 5.2 \\
\hline \multirow[t]{4}{*}{$\Delta \epsilon / \epsilon$} & A & -1.10 & -1.19 & -1.22 & -1.12 & -1.24 & -1.25 \\
\hline & B & -1.02 & -1.13 & -1.17 & -1.24 & -1.18 & -1.05 \\
\hline & $\mathrm{C}$ & -0.92 & -1.04 & -1.02 & -1.11 & -0.96 & -1.06 \\
\hline & D & -1.16 & -1.19 & -1.20 & -1.29 & -1.17 & -1.27 \\
\hline$A_{1}$ & A calc & 2.7 & 8.5 & 12 & 13 & 27 & 35 \\
\hline \multirow[t]{7}{*}{$(\%)$} & $A \exp$ & - & - & 10 & 14 & 31 & 40 \\
\hline & B calc & 3.8 & 9.9 & 13 & 15 & 30 & 39 \\
\hline & $B \exp$ & - & 10 & 14 & 16 & 28 & 40 \\
\hline & $\mathrm{C}$ calc & 5.7 & 14 & 17 & 21 & 42 & 50 \\
\hline & $C \exp$ & - & 10 & 16 & 18 & 33 & 50 \\
\hline & $\mathrm{D}$ calc & 0.7 & 3.1 & 4.5 & 5.6 & 13 & 18 \\
\hline & $D \exp$ & - & - & 10 & 14 & 30 & 40 \\
\hline
\end{tabular}

${ }_{\alpha}^{\text {a }}$ Polarizability in $\left[10^{-33} \mathrm{Cm}^{2} \mathrm{~V}^{-1}\right] ; E_{0}$ saturation field strength in $[\mathrm{kV} / \mathrm{cm}] ; \Delta \epsilon / \epsilon$ reduced electric dichroism in the limit of complete orientation and complete stretching; $A_{1}$ relative bending amplitudes: the calculated values result from the orientation function (cf. text) and the experimental ones from the fast relaxation component of the dichroism decay curves. The data have been obtained in buffers containing $1 \mathrm{~m} \mathrm{M} \mathrm{NaCl}, 1 \mathrm{~m} M \mathrm{Na}$-cacodylate, pH 7.0; additional components are (A) $100 \mathrm{mM} \mathrm{MgCl}$; (B) $100 \mu M$ EDTA, $3 \mu M$ spermine, (C) $10 \mu M\left[\mathrm{Co}\left(\mathrm{NH}_{3}\right)_{6}\right]^{3+}$; (D) $100 \mu M$ EDTA. The persistence lengths $p$ used for the model calculations $\operatorname{are}^{13}$ (A) $350 \AA$, (B) $330 \AA,(C) 200 \AA$, and (D) $700 \AA$. Estimated relative accuracies are $\alpha \pm 10 \%, E_{0} \pm 20 \%$, $\Delta \epsilon / \epsilon \pm 10 \%, A_{1} \pm 20 \%$; all data at $20^{\circ} \mathrm{C}$.

short DNA fragments. The advantage of this approximation is that it is relatively easily tractable and it can be extended without much difficulty to the case of stably curved DNA. More general models should be developed for long DNA chains, but such models clearly require much more extensive computations than the present model.

It should be mentioned that in a previous investigation Jernigan and Miyazawa ${ }^{26}$ discussed Kerr effects of flexible macromolecules. However, their goals and their approach were clearly different from the ones pursued in the present study.

Evaluations of experimental data by the new orientation function requires extensive computations, which is not a serious disadvantage anymore owing to the availability of increasingly efficient computational facilities. In this context it should be noted that quantitive evaluations of experimental data according to a "simple" induced dipole model also requires numerical integrations. One advantage of the numerical approach is the possibility of modifying 
functions readily without the need to select expressions that can be solved analytically. An obvious extension of the present model is the consideration of stable curvature, for example.

In a comparison of the predictions given by the present orientation function with experimental results one has to bear in mind that some effects induced by application of electric field pulses have not been considered. One of these effects is electrophoresis of molecules with a net charge, which will lead to some alignment of rodlike molecules due to hydrodynamic coupling. Because it is rather difficult to model the hydrodynamics properly, at present there is not even a reasonable estimate of its influence on the degree of orientation. Any contribution resulting from this effect will lead to some error in the model parameters - a problem common to all available orientation functions.

Another problem exists with respect to the assignment of experimental bending amplitudes. The rotational diffusion of particles without symmetry is reflected in electrooptical experiments by 5 exponentials. ${ }^{27}$ In the case of straight DNA double helices, this is reduced to a single exponential due to symmetry. However, bending of the double helix into a circular arc leads to the appearance of a second exponential. Model simulations ${ }^{21}$ show that the amplitude of this additional exponential remains very small, unless the DNA is bent to a considerable extent. Thus, the present assignment of bending amplitudes is expected to be correct in the limit of relatively low chain lengths. A clear indication for a correct assignment of experimental bending amplitudes comes from the fact that these amplitudes are very small at low field strengths and increase to a considerable extent with increasing field strength. This observation is hardly consistent with any dominant contribution to the fast amplitude due to constant stable curvature and demonstrates that there is a considerable field-induced change in the bending distribution.

The comparison of the experimental data with the model reveals a surprisingly close agreement for all buffer conditions with the exception of the buffer, which contains monovalent ions only. In the latter case the bending amplitudes predicted by the model are much lower than those found from the dichroism decay curves. According to the present model, the magnitude of the bending amplitudes is determined mainly by the persistence length: at given values of the chain length and the electric field strength the amplitudes increase with decreasing persistence length. In the previous investigation ${ }^{13}$ of bending amplitudes it had been already demonstrated that the expected relationship between bending amplitude and persistence length does not always hold. With the results of the present, more quantitative model analysis, the origin of the discrepancy may be discussed in more detail.

First of all, it remains possible that the present model is not sufficiently accurate. However, the satisfactory agreement observed for both the chain length and the field strength dependence in three different buffers indicates that the most important field-induced processes have been described with sufficient accuracy.

The deviations happen to be observed for an ion composition, where DNA double helices have a much lower (thermal) stability compared to that given in the other three buffers. Thus it may be suspected that some field-induced conformation change is responsible for the deviation. Although there is no field-induced absorbance change at the magic angle or any other indication in 
favor of this interpretation, a subtle field-induced conformation change cannot be ruled out completely.

A more interesting explanation, which is also more likely at the present state of knowledge, may be given in terms of buffer-dependent contributions from stably curved DNA sequences. ${ }^{13}$ The present investigation shows that the bending amplitudes observed in the presence of $\mathrm{Mg}^{2+}$, spermine, and even $\left[\left(\mathrm{Co}\left(\mathrm{NH}_{3}\right)_{6}\right]^{3+}\right.$ can be accounted for by the experimental persistence length without explicit consideration of curvature. It should be noted, however, that the experimental persistence length reflects both thermal bending and stable curvature. Thus, it remains possible that the long-range structure indicated by the persistence length in the presence of $\mathrm{Mg}^{2+}$, spermine, or $\left[\mathrm{Co}\left(\mathrm{NH}_{3}\right)_{6}\right]^{3+}$ is not only due to thermal bending but also due to some stable curvature. ${ }^{28}$ Recently separation of these effects has been attempted on the basis of models derived from gel mobilities and enzymatic circularization probabilities. ${ }^{29}$ Separation on a more direct biophysical basis proves to be difficult. In a recent electrooptical investigation ${ }^{30}$ a DNA fragment, which should be strongly curved according to its gel mobility, exhibited rotational diffusion time constants, which are very similar to those of "straight" DNA fragments. These results raise serious questions on the properties of "curved" DNA molecules in solution. A further analysis should be possible on the basis of the present orientation function for flexible molecules, which may be extended to include curvature. For this purpose it will be important again to use an appropriate function to describe stretching of molecules in electric fields. The present results demonstrate that the stretching process can be described reasonably well by the dipole forces, which cause orientation. In addition, it has been demonstrated that the bending distribution strongly affects stationary electrooptical data of flexible molecules and thus should not be neglected in any quantitative evaluation of such data. Since most polymers are flexible at least to some degree, the present orientation function should not only be useful for the analysis of DNA molecules but also for the investigation of other polymers.

The author is greatly indebted to Gerald Manning for discussions and comments on mechanical models of DNA structure. Thanks are due to Jan Antosiewicz for reading the manuscript. Numerical results were calculated on the facilities of the Gesellschaft für wissenschaftliche Datenverarbeitung m.b.H, Göttingen.

\section{References}

1. Fredericque, E. \& Houssier, C. (1973) Electric Dichroism and Electric Birefringence, Clarendon Press, Oxford.

2. O'Konski, C. T. (1976) Molecular Electro-Optics, Dekker, New York.

3. Charney, E. (1988) Quart. Rev. Biophys. 21, 1-60.

4. Hogan, M., Dattagupta, N. \& Crothers, D. M. (1978) Proc. Natl. Acad. Sci. USA 75, 195.

5. Diekmann, S., Hillen, W., Jung, M., Wells, R. D. \& Porschke, D. (1982) Biophys. Chem. 15, $157-167$.

6. Stellwagen, N. C. (1981) Biopolymers 30, 399.

7. Elias, J. G. \& Eden, D. (1981) Macromolecules 14, 410.

8. Hagermann, P. J. (1981) Biopolymers 20, 1503.

9. Diekmann, S., Hillen, W., Morgeneyer, B., Wells, R. D. \& Porschke, D. (1982) Biophys. Chem. 15, 263-270.

10. Landau, L. D. \& Lifshitz, E. M. (1958) Statistical Physics, Pergamon Press, London. 
11. Bloomfield, V. A., Crothers, D. M. \& Tinoco, I., Jr. (1974) Physical Chemistry of Nucleic Acids, Harper \& Row, New York.

12. Manning, G. (1985) Cell Biophys. 7, 57-89.

13. Porschke, D. (1986) J. Biomol. Struct. Dynam. 4, 373-389.

14. Kam, Z., Borochov, N. \& Eisenberg, H. (1981) Biopolymers 20, 2671-2690.

15. Borochov, N. \& Eisenberg, H. (1984) Biopolymers 23, 1757-1769.

16. Mandel, M. (1961) Mol. Phys. 4, 489-496.

17. Fixman, M. \& Jagannathan, S. (1981) J. Chem. Phys. 75, 4048-4059.

18. Mandel, M. \& Odijk, T. (1984) Ann. Rev. Phys. Chem. 35, 75.

19. Porschke, D. (1985) Biophys. Chem. 22, 237-247.

20. Saenger, W. (1983) Principles of Nucleic Acid Structure, Springer, Berlin.

21. Antosiewicz, J. \& Porschke, D., Biophys. Chem., in press.

22. Yoshioka, K. (1983) J. Chem. Phys. 79, 3482-3486.

23. Diekmann, S., Jung, M. \& Teubner, M. (1984) J. Chem. Phys. 80, 1259-1262.

24. Hearst, J. E. (1963) J. Chem. Phys. 38, 1062-1065.

25. Hagermann, P. J. (1988) Ann. Rev. Biophys. Chem. 17, 265-286.

26. Jernigan, R. L. \& Miyazawa, S. (1981) in Molecular Electro-Optics, Krause, S., Ed., Plenum Press, New York.

27. Wegener, W. A., Dowben, R. M. \& Koester, V. J. (1979) J. Chem. Phys. 70, 622-632.

28. Trifonov, E. N. (1985) CRC Crit. Rev. Biochem. 19, 89-106.

29. Trifonov, E. N., Tan, R. K. Z. \& Harvey, S. C. (1987) in DNA Bending and Curvature, Olson, W. K., Sarma, M. H., Sarma, R. H., \& Sundaralingam, M., Eds., Adenine Press, New York. 30. Diekmann, S. \& Porschke, D. (1987) Biophys. Chem. 26, 207-216.

Received July 6, 1988

Accepted September 22, 1988 